\title{
Electric-Field-Controlled Synthesis of HPMA Hydrogels Containing Self-Organized Arrays of Micro-Channels
}

\author{
TEDRIC D. CAMPBELL, RANDY P. WASHINGTON, OLIVER STEINBOCK \\ Department of Chemistry and Biochemistry, Florida State University, Tallahassee, Florida 32306-4390
}

Received 8 December 2006; accepted 10 February 2007

DOI: 10.1002 / pola.22016

Published online in Wiley InterScience (www.interscience.wiley.com).

\begin{abstract}
We report the synthesis of poly $N$-(2-hydroxypropyl)methacrylamide ordered arrays of fluid filled channels. The polymerization and crosslinking reactions are carried out under the influence of a constant electric field $(60 \mathrm{~V} / \mathrm{cm})$. A charged comonomer, immobiline (pK 3.6), and porogen, polyethylene glycol (PEG) are added to the pregel solutions. Scanning electron microscopy reveals that the channels have a typical diameter of $2-25 \mu \mathrm{m}$ and are oriented parallel to the electric field employed during synthesis. The self-organization of channels occurs around an optimal PEG concentration of $8.6 \mathrm{wt} / \mathrm{vol} \%$, whereas significantly higher or lower concentrations yield random, isotropic pore structures. Moreover, tensile strength measurements show that the mechanical stability increases with decreasing concentration of PEG. Rheology experiments reveal that the swelling degree of these superabsorbant hydrogels increases with increasing PEG. Possible applications of these microstructured hydrogels as bidirectional scaffolds for regenerating neurons in the injured spinal cord are discussed. $\odot 2007$ Wiley Periodicals, Inc. J Polym Sci Part A: Polym Chem 45: 2593-2600, 2007
\end{abstract}

Keywords: biomaterials; hydrogels; morphology; nerve conduits; P-HPMA hydrogel; swelling

\section{INTRODUCTION}

Hydrogels are three-dimensional, sponge-like networks of crosslinked polymers with high porosity and large internal surface area. Crosslinking can be chemical, due to covalent bonds, or physical, due to hydrogen bonding, van der Waals interactions, or physical entanglements. ${ }^{1}$ If the polymer network is able to swell to over $95 \%$ of its dry volume, then it can be classified as a superabsorbant. ${ }^{2}$ Hydrogels can swell or shrink in response to external stimuli such as $\mathrm{pH}$, ionic strength, and temperature. ${ }^{3-6}$ This feature is closely related to the hydrophilicity of the polymer chains and the crosslink density. These prop-

Correspondence to: O. Steinbock (E-mail: steinbck@chem. fsu.edu)

Journal of Polymer Science: Part A: Polymer Chemistry, Vol. 45, 2593-2600 (2007) @2007 Wiley Periodicals, Inc. erties have been modified for numerous applications in biomedicine, pharmaceutics, bioengineering, and neurology. ${ }^{7,8}$

The swelling behavior of hydrogels depend on the physicochemical characteristics of the external and internal polymer surfaces. Accordingly, there is a great need for techniques that tailor the properties of polymer surfaces to enhance their usefulness. ${ }^{9}$ For example, it has been shown that hydrogels can be employed as templates to create mesoporous silica monolith containing oriented macroporous channels. ${ }^{10}$ The latter study demonstrates that surface modification of polymers not only include surface chemistry but polymer morphology as well. However, hydrogels are typically created with a wide range of pore sizes that promotes irregularity in structure. This feature can limit the hydrogel effectiveness as templates, especially for certain biological applications. 
A class of polymers based on the monomer $N$ (2-hydroxypropyl) methacrylamide (HPMA) is of particular current interest because of its versatile chemistry and good biocompatibility. Prior studies on copolymers of HPMA have shown the effect of ionogenic groups on the permeability and sorption capacity of polymer networks. ${ }^{11}$ Polymers based on HPMA have also been examined as water-soluble anticancer drug carriers. ${ }^{12}$ With its many uses, HPMA appears to be an ideal monomer for hydrogel applications in biomedicine and specifically neuronal regeneration. ${ }^{13}$

The goal of the present study is the preparation of poly $N$-(2-hydroxypropyl)methacrylamide (PHPMA) hydrogels in which the typically random polymer network is altered to arrays of parallel channels. The study is motivated in part by the idea to implant hydrogels as bridge materials that promote uniaxial regenerative growth in the damaged central nervous system such as the injured spinal cord. ${ }^{14}$ For this purpose, the diameter of the channels should match the archetypal diameter of neuronal cell bodies or their axons to provide an optimal internal surface structure. With that aim in mind, we devise and optimize an experimental technique in which a modified HPMA-pregel solution is polymerized and crosslinked in the presence of an externally applied electric field. In addition, the effects of polyethylene glycol (PEG) on the efficacy of channel formation, swelling dynamics, and the mechanical characteristics of the resulting material are investigated.

\section{EXPERIMENTAL}

\section{Materials}

The crosslinker $N, N^{\prime}$-methylenebisacrylamide (Bis, Fluka), the initiator ammonium persulfate (APS, Fluka), and the accelerator $N, N, N^{\prime}, N^{\prime}$ tetramethylethylenediamine (TEMED, Aldrich) were procured and used as received. Immobiline pK 3.6 ( $N$-acryloyl glycine), tris(hydroxymethyl) aminomethane (Tris), and glycine were obtained from Pharmacia Biotech. PEG, $10 \mathrm{kDa}$, (Fluka) was used as a porogenic agent. Methacryloyl chloride and 1-amino-2-propanol, both from Aldrich, and solvents methylene chloride and acetone, both from Fisher, were used without further purification for the synthesis of the HPMA monomer.

\section{Synthesis of HPMA Monomer}

The radical polymerization of HPMA has previously been discussed. ${ }^{15}$ The monomer was pre- pared from the reaction between methacryloyl chloride and 1-amino-2-propanol in dry organic solvent in the presence of the acid acceptor sodium bicarbonate. A well-stirred mixture of 1amino-2-propanol $(0.30 \mathrm{~mol})$ in methylene chloride $(85 \mathrm{~mL})$ cooled to $0{ }^{\circ} \mathrm{C}$ was supplemented with $33.6 \mathrm{~g}$ of anhydrous sodium bicarbonate $(0.34 \mathrm{~mol})$ and $0.25 \mathrm{~g}$ of hydroquinone. Methacryloyl chloride $(0.29 \mathrm{~mol})$ in $40 \mathrm{~mL}$ of methylene chloride was then added drop wise over a $1 \mathrm{~h}$ period and the reaction mixture was then stirred at $15{ }^{\circ} \mathrm{C}$ for an additional hour. Sodium sulfate $(10 \mathrm{~g})$ was added to the solution and after mixing for $1 \mathrm{~h}$, the excess solid consisting of sodium bicarbonate and sodium sulfate was removed by vacuum filtration. The monomer was purified by triple recrystallization from acetone. The synthesis of the monomer leads to a percent yield of $62 \%$. The melting point was measured to be $69{ }^{\circ} \mathrm{C}$, comparable to reported values. ${ }^{16,17}$

Carbon and nitrogen elemental analysis was performed on the HPMA crystals using a ThermoQuest analyzer (Model: EA/NA 1110 with Eager 200 data acquisition). The mass percentage found for nitrogen and carbon was 9.53 and $57.96 \%$, respectively. The latter values are in good agreement with the calculated mass percentages (9.78\% for nitrogen and $58.76 \%$ for carbon).

${ }^{1} \mathrm{H}$ and ${ }^{13} \mathrm{C}-\mathrm{NMR}$ spectra were recorded in $\mathrm{CDCl}_{3}$ at concentrations of $100 \mathrm{~g} / \mathrm{L}$ on a Bruker ARX spectrometer, operating at 200 and 75.4 $\mathrm{MHz}$, respectively. The results indicate that the synthesis yielded a pure product. The chemical shifts $\delta$ (ppm) of ${ }^{1} \mathrm{H}-\mathrm{NMR}$ are:

(a) $1.14-1.16\left(\mathrm{~d}, \mathrm{CH}_{3}\right),(\mathrm{b}) 1.92\left(\mathrm{~s}, \mathrm{CH}_{3}\right),(\mathrm{c})$ 3.08-3.17 (st, $\mathrm{CH}_{2}$ ) (d) 3.29 (s, OH), (e) 3.41-3.49 (ot, $\mathrm{CH}_{2}$ ), (f) 3.85-3.95 (dt, $\mathrm{CH}$ ), (g) $5.30\left(\mathrm{~s}, \mathrm{CH}_{2}\right.$ ), (h) $5.69\left(\mathrm{~s}, \mathrm{CH}_{2}\right)$, and (i) $6.50(\mathrm{~s}, \mathrm{NH})$. The chemical shifts of ${ }^{13} \mathrm{C}-\mathrm{NMR}$ are: (a) $18.57\left(\mathrm{CH}_{3}\right)$, (b) $20.87\left(\mathrm{CH}_{3}\right)$, (c) $47.09\left(\mathrm{CH}_{2}\right)$, (d) 67.17 (C), (e) $119.99\left(\mathrm{CH}_{2}\right)$, (f) $139.57(\mathrm{CH})$, and $(\mathrm{g}) 169.39(\mathrm{CO})$.

\section{Polymerization at Constant Voltage}

A FisherBiotech vertical electrophoresis unit (FBVE10-1) was used to synthesize the hydrogels under a constant electric field. The procedure is similar to the one reported by Charlionet et al. for the synthesis of oriented polyacrylamide gels for quick analysis of proteins and DNA. ${ }^{18}$ The upper buffer solution consisted of $200 \mathrm{mM}$ Tris and $50 \mathrm{mM}$ glycine supplemented with $20 \mathrm{mM}$ APS, while the lower buffer solution consisted of $200 \mathrm{mM}$ Tris and $50 \mathrm{mM}$ glycine. For the synthe- 
sis of P-HPMA hydrogel, the following substances were added sequentially into a vial: $0.50 \mathrm{~g}$ HPMA, $75 \mathrm{mg} N, N^{\prime}$-methylenebisacrylamide, $0.50 \mathrm{~mL}$ of immobiline $(0.20 \mathrm{M}$ stock solution), $0.5 \mathrm{~g}$ PEG, and $4.75 \mathrm{~mL}$ of distilled water. The freshly prepared solution was deoxygenated by purging with nitrogen for $5 \mathrm{~min}$. Following the addition of $4.9 \mathrm{mg}$ of APS and $2 \mu \mathrm{L}$ of TEMED to the pregel solution and rapid mixing by swirling, the solution was transferred between two $10 \mathrm{~cm}$ $\times 10 \mathrm{~cm}$, previously silanized, glass plates separated by $3-\mathrm{mm}$ teflon spacers. The system was sealed at the bottom by a $1.5 \%$ agarose gel (height $\sim 3 \mathrm{~cm}$ ). The agarose base included $20 \%$ lower buffer solution and $20 \mu \mathrm{L}$ TEMED. A power supply (Bio-Rad, Powerpac 3000) was used to provide a constant voltage of $60 \mathrm{~V} / \mathrm{cm}$ at typically $10 \mathrm{~mA}$. Once TEMED was added, the polymerization reaction commenced with voltage being applied immediately ( $\sim$ time delay $<5 \mathrm{~s}$ ). The hydrogel formed within $30 \mathrm{~min}$; however, the system was left undisturbed for an additional hour to guarantee optimal monomer conversion. ${ }^{19}$

\section{Cryosectioning}

A cryostat, Microm Model HM 500 M, was used to obtain slices of frozen hydrogel samples. A 6.50 $\mathrm{cm}^{3}$-block hydrogel sample was wrapped in aluminum foil and placed in a beaker containing 2methylbutane. The beaker was then submerged in liquid nitrogen for $30 \mathrm{~s}$. The frozen hydrogel was attached to the cryostat plate using tissue freezing medium and cut at $25 \mu \mathrm{m}$.

\section{Morphology}

The morphology of P-HPMA hydrogel was examined with a scanning electron microscope (JEOL 5900). For these measurements, the samples were placed in $30 \%$ sucrose and freeze-dried overnight. The samples were then sputtered with gold and imaged using an accelerating voltage of $5 \mathrm{kV}$.

\section{Tensile Properties}

Uniaxial load-strain experiments were carried out using a Thermomechanical Analyzer (TA Instruments, 4950) at room temperature in the stress-strain mode. The tests were performed on swollen sample to measure the effect of the porosity of the matrix on the mechanical properties. The apparent Young's modulus was obtained from the initial slope of the individual stress-versusstrain plots.

\section{Swelling Kinetics}

The swelling properties were examined with an analytical balance (Denver Instrument Company M-220) and a peristaltic pump (Dynamax RP-1). The experiments were conducted at room temperature. The fully swollen hydrogels were weighed, placed in conical vials, submerged in liquid nitrogen $(\approx 10 \mathrm{~s})$, and then dehydrated using a speed vacuum concentrator. Ultrapure water was pumped onto the dried hydrogel for a certain time interval after which the sample was reweighed. Swelling experiments were carried out on the dried hydrogels until the original weight was obtained. All experiments were performed in duplicate. The flow rate was kept constant at 0.33 $\mathrm{mL} / \mathrm{min}$. The swelling percent is defined as:

$$
\text { Swelling } \%=\frac{\left(W_{\mathrm{s}}-W_{\mathrm{d}}\right)}{W_{\mathrm{d}}} \times 100 \%
$$

where $W_{\mathrm{s}}$ is the weight of the swollen sample and $W_{d}$ is the weight of the dry sample.

\section{RESULTS AND DISCUSSION}

\section{Hydrogel Synthesis}

The different feed compositions and the properties of the resulting hydrogels are summarized in Table 1 . The hydrogels synthesized with PEG are slightly translucent with no obvious opaque domains in the matrix, indicating that the three dimensional structure is microporous. As the PEG concentration is increased, the resulting hydrogels become opaque probably owing to phase separation of the HPMA polymer chains and the PEG molecules. PEG is known to elicit macroporosity in polyacrylamide and poly( $N$-isopropyl acrylamide) hydrogels through similar hydrogen bonding interaction. ${ }^{20,21}$

\section{Characterization by Microscopy}

The scanning electron micrographs of the surfaces of hydrogels synthesized with and without applied electric field showed marked differences in their three-dimensional architecture. The characteristic surface morphology of the dried hydrogel samples is illustrated in Figure 1 which 
Table 1. Final Composition and Properties of the Analyzed P-HPMA Hydrogels

\begin{tabular}{cccc}
\hline Sample & $\begin{array}{c}\text { PEG } \\
(\text { wt/vol \%) }\end{array}$ & $\begin{array}{c}\text { Appearance of } \\
\text { Hydrogel }\end{array}$ & $\begin{array}{c}\text { Statement of } \\
\text { Orientation }\end{array}$ \\
\hline H1 & 0.0 & Slightly translucent & No alignment \\
H2 & 4.8 & Slightly opaque & Partial alignment \\
H3 & 8.6 & Opaque & Aligned \\
H4 & 9.5 & Opaque & Partial alignment \\
H5 & 14.2 & Opaque & No alignment \\
H6 & 19.0 & Opaque & No alignment \\
\hline
\end{tabular}

represents H3. Comparing the two micrographs, it is evident that the morphology of the hydrogel without an electric field shows a seemingly isotropic and nonpatterned material. The hydrogel prepared under the influence of an electric field, however, reveals a stratified structure interconnected through a porous network. This stratification is indicative of the existence of a highly ordered, three-dimensional array of parallel channels. Within the swollen hydrogel, these channels are filled with solvent and are partitioned by aggregates of crosslinked polymer fibers. Further inspection revealed that the stratification extends through the entire sample, although some pronounced defects were observed that we interpret to be artifacts created during the cutting and fracturing of the gel. From samples synthesized under this set of experimental conditions, we



determined the typical width of the channels as $2.5 \mu \mathrm{m}$.

To assess the orientation of the channels with respect to the applied electric field, optical micrographs of the specimen were obtained for hydrogels with formulation similar to H3. Experiments employed a Leica DMIRB microscope. The samples were cut and handled in a way that allowed us to relate the structure of the hydrogels to the direction of the electric field. Under bright-field illumination, faint stripes were observed within the samples. Their orientation revealed that the fluid-filled channels in the hydrogel align parallel to the electric field applied during synthesis. The same conclusion was drawn from bright-filled micrographs of $25 \mu \mathrm{m}$ cryosectioned gel slices (Fig. 2).

It appears likely that the charged immobiline molecules are driven towards the cathode and

Figure 1. Representative scanning electron micrographs of hydrogel H3 synthesized (a) with electric field $(60 \mathrm{~V} / \mathrm{cm})$ and (b) without electric field. A random network that forms an overall microporous material is characteristic of samples synthesized without electric field, whereas the hydrogel prepared under the influence of an electric field reveals a stratified surface interconnected through a porous network. The $2.5-\mu \mathrm{m}$ channels, stemming from the proposed alignment of immobiline molecules, are oriented parallel to the applied electric field. Field of view: $(60 \times 80) \mu \mathrm{m}^{2}$. 




Figure 2. Bright-field micrograph of a $25-\mu \mathrm{m}$ thick cryosectioned HPMA hydrogel. Scale bar is $10 \mu \mathrm{m}$. The inset shows the corresponding two-dimensional Fourier transform. Field of view: $(165 \times 125) \mu \mathrm{m}^{2}$.

therefore align covalently-bound polymer chains along the electric field lines. These microscopic dynamics seem to perturb the subsequent phase separation process in a symmetry-breaking fashion and induce the formation of mesoscopic channels. This nonequilibrium pattern is subsequently fossilized by the bisacrylamide induced crosslinking of polymer chains. To test this hypothesis, we performed a series of electric-fieldcontrolled syntheses in the absence of immobiline. The results revealed no indications of channels or any preferential alignment and the corresponding microstructure was randomly oriented over the intertwined microporous network. These observations emphasize that the charged comonomer, immobiline, is indeed crucial for the formation of channels and, hence, support our original interpretation.

We also find that the existence of channels is strongly dependent on the concentration of the hydrophilic moiety PEG. Scanning electron microscopy (SEM) inspection of the fractured surface revealed that PEG substantially affects the presence of oriented channels as shown in Figure 3(a-f). The highest degree of order within the channel arrays is found at an optimal PEG concentration of around $8.6 \mathrm{wt} / \mathrm{vol} \%$ as illustrated in Figure 3(c). In this case, the 2.5- $\mu \mathrm{m}$ tubes are well aligned along the electric field direction and only a small number of defects are observed in the stripe pattern of the fractured specimen.

Partially aligned channels in P-HPMA can be obtained under an applied electric field if PEG is present in a range slightly above or below the op- timum PEG content. Figure 3(b,d) shows the micrometer-scale structure of hydrogels that were synthesized in this concentration regime. Here, the channels are also oriented in the direction of the electric field, but the patterns tend to be more erratic and reveal a larger defect density. Moreover, variations of the PEG concentrations below and above 8.6 wt \% result in hydrogels with larger channel diameters. For lower concentrations, their width varies between 5 and $10 \mu \mathrm{m}$ [e.g., Fig. 3(b)], whereas hydrogels formulated with slightly higher concentration of PEG are filled with 20-25 $\mu$ m channels [e.g., Fig. 3(d)].

The materials prepared at high levels of PEG have a larger population of micro- and macropores. In the $14.2 \mathrm{wt} / \mathrm{vol} \%$ PEG samples examined, bundles of tightly packed longitudinally arranged microfibrils permeate throughout the structured polymer matrix [Fig. 3(e)]. These microfibrils still have a preferential orientation in the direction of the applied electric field. However, as shown in Figure 3(f), a further increase in PEG concentration to $19.0 \mathrm{wt} / \mathrm{vol} \%$ randomizes the orientation of channels, resulting in an open porous network with an average pore size of $\sim 5 \mu \mathrm{m}$. Additionally, we find that channels do not exist in samples prepared in the absence of PEG. Under these conditions, hydrogels show a uniform microporous network with an interconnected pore morphology and no global orientation. A typical example for these isotropic hydrogels is shown in Figure 3(a).

\section{Elastic Properties}

The mechanical integrity of our P-HPMA hydrogels was evaluated by determining Young's moduli of hydrogels synthesized at various PEG concentrations. The rigidity of the cavity walls, and consequently the elastic modulus, is likely related to the nature of interaction of the monomers and PEG during synthesis. Since the pore size is strongly influenced by the hydrophilic PEG, swelling capacity should increase with increasing PEG concentration and be accompanied by a decrease in structural integrity. Thus, a higher mechanical stability is expected for hydrogels formed at lower PEG concentrations because of the hydrophilicity under these conditions. Figure 4 summarizes the elastic properties as a function of PEG concentration. The data reveal a decrease in mechanical strength for increasing PEG concentration and obey a nearly linear dependence in the $8.6-19.0 \mathrm{wt} / \mathrm{vol} \%$ regime. The decrease in 

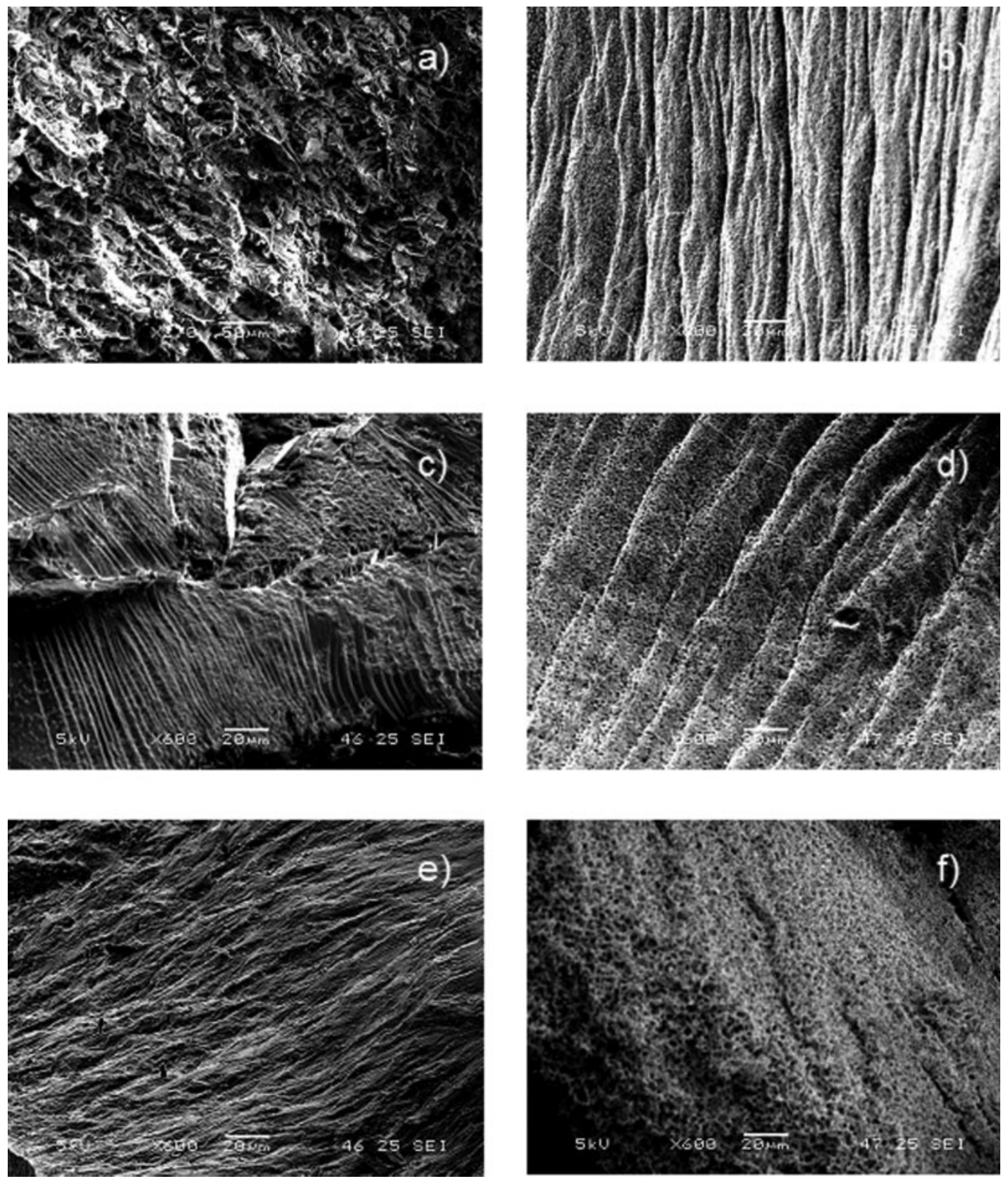

Figure 3. Micrographs of hydrogels H1-H6 showing the efficacy of channels formation at different concentration of PEG: (a) 0.0, (b) 4.8, (c) 8.6, (d) 9.5, (e) 14.2, and (f) $19.0 \mathrm{wt} / \mathrm{vol} \%$. The morphologies of these hydrogels range from a microporous network to aligned channels at an optimal concentration of PEG, noted for sample H3 $(8.6 \mathrm{wt} / \mathrm{vol}$ $\%)$. Field of view: $(120 \times 160) \mu \mathrm{m}^{2}$. 


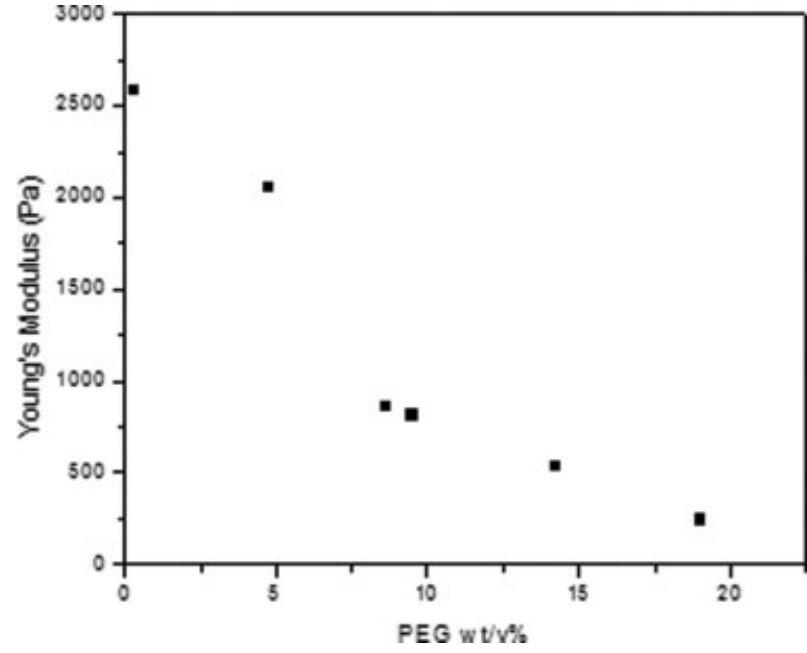

Figure 4. Young's modulus of swollen hydrogels at $25{ }^{\circ} \mathrm{C}$ for various PEG concentrations. The elastic modulus is determined from the initial slopes of stressstrain curves.

Young's modulus with increasing concentration is in accord with the SEM observation that the porosity of the hydrogels increases with PEG.

\section{Swelling Properties}

Study of the HPMA based hydrogels led to the following results. The percent swelling capacity increased as PEG wt/vol \% increased. As seen in Figure 3, an increase in PEG decreases channel efficiency, but increases the porosity of the hydrogel. This pore size increase allows for shorter swelling times. Figure 5 summarizes the swelling



Figure 5. Time difference of the swelling behavior of hydrogels in ultrapure water for PEG wt/vol \% of 0.0 , 8.6, and 19.0 .

Journal of Polymer Science: Part A: Polymer Chemistry DOI 10.1002/pola


Figure 6. Image of H3 in (a) dry state and (b) swollen state. [Color figure can be viewed in the online issue, which is available at www.interscience.wiley.com.]

percent as a function of time. The 19.0 PEG wt/ vol \% swelled to $39 \%$ after initial contact with solvent, compared to $26 \%$ and $19 \%$, for PEG wt/vol \% 0.0 and 8.6, respectively.

It should be noted that the short swelling times are affected by the nature of dehydration and the flow rate of the peristaltic pump. When drying, each sample lost $92 \%$ of its weight, but because it was first submerged in liquid nitrogen, the dimensions were similar (Fig. 6). This is also due to the hydrogel's ability to maintain strong mechanical properties at high swelling ratios. ${ }^{22}$ Each sample took less than $1 \mathrm{~mL}$ of $\mathrm{H}_{2} \mathrm{O}$ to reach its original weight.

\section{CONCLUSIONS}

In conclusion, we have demonstrated the synthesis of P-HPMA hydrogels that contain highly ordered arrays of fluid-filled channels. To obtain these oriented patterns in the macroporous hydrogels, the electric-field alignment of immobiline (pK 3.6) must be fossilized by polymerization and crosslinking reactions. The efficacy of channel formation is strongly affected by the presence of PEG, with the optimal self-organization of channels occurring around a PEG concentration of $8.6 \mathrm{wt} / \mathrm{vol} \%$.

Microscopic analyses revealed that the channels have a typical diameter of $2-25 \mu \mathrm{m}$ and are oriented parallel to the applied electric field. With the diameter of these channels coinciding with the typical length scales of neuronal cell bodies and axons, these striated specialty materials could be used as porous biocompatible scaffolds for regenerating neurons in the injured spinal cord.

Further experiments are being conducted using these and other synthetic hydrogels with 
variable microstructure and funtionalization to determine the scope and potential of electric field alignment of monomers as a novel method to produce micropatterned hydrogels channels. Research in the preparation of specialty materials based on 2-hydroxyethylmethacrylate that contain well aligned microchannels has already begun, but it remains to be seen if the methodology is readily transferable to other hydrogels system, particularly the interesting HPMA-RGD (Neurogel $\left.{ }^{\mathrm{TM}}\right) \cdot{ }^{23-25}$ Insights into the alignment of the polymerizable RGD chains by an electric field, and their subsequent relative reactivity, should allow the systematic variation of the gel structure to produce hydrogels with tailored channel dimensions and optimal mechanical properties.

The authors thank B. T. Ginn for help during the initial stage of this investigation. This work was partially supported by the National Science Foundation under Grant No. 0513912 and the Petroleum Research Fund (ACS-PRF).

\section{REFERENCES AND NOTES}

1. Kamath, K.; Park, K. Adv Drug Delivery Rev 1993, 11, 59.

2. Park, H.; Park, K. In Hydrogels and Biodegradable Polymers for Bioapplications; Ottenbrite, R. M.; Huang, S. J.; Park, K., Eds.; American Chemical Society: Washington, DC, 1996; p 2.

3. Ju, H. K.; Kim, S. Y.; Lee, Y. M. Polymer 2001, 42, 6851.

4. Paneva, D.; Mespouille, L.; Manolova, N.; Degee, P.; Rashkov, I.; Dubois, P. J Polym Sci Part A: Polym Chem 2006, 44, 5468.

5. Zhang, R.; Tang, M.; Bowyer, A.; Eisenthal, R.; Hubble, J. Biomaterials 2005, 6, 4677.

6. Xu, F. J.; Kang, E. T.; Neoh, K. G. Biomaterials 2006, 27, 2787.

7. Gupta, P.; Vermani, K.; Garg, S. Drug Discov Today 2002, 7, 569.
8. Donini, D.; Robinson, N.; Colombo, P.; Giordano, F.; Peppas, N. A. Int J Pharm 2002, 245, 83.

9. Santiago, L. Y.; Nowak, R. W.; Rubin, J. P.; Marra, K. G. Biomaterials 2006, 27, 2962.

10. Giunta, P. R.; Washington, R. P.; Campbell, T. D.; Steinbock, O.; Stiegman, A. E. Angew Chem Int Ed 2004, 43, 1505.

11. Denim, A. A.; Melenevskii, A. T.; Ochkur, O. V.; Shkol'nikov, E. I.; Sidorova, E. V.; Sidorov, A. A.; Gromadkova, I. Russ J Phys Chem 2006, 80, 1484.

12. Sykova, E.; Mazel, T.; Vargova, L.; Vorisek, I.; Prokopova-Kubinova, S. Prog Brain Res 2000, 125, 155.

13. Hong, C. Y.; You, Y. Z.; Pan, C. Y. J Polym Sci Part A: Polym Chem 2006, 44, 2419.

14. Tian, W. M.; Zhang, C. L.; Hou, S. P.; Yu, X.; Cui, F. Z.; Xu, Q. Y.; Sheng, S. L.; Cui, H.; Li, H. D. J Controlled Release 2005, 102, 13.

15. Kopeîcek, J.; Bazilová, H. Eur Polym J 1973, 9, 7.

16. Ulbrich, K.; Subr, V.; Stroholm, J.; Plocova, D.; Jelinkova, M.; Richova, B. J Controlled Release $2000,64,63$.

17. Strohalm J.; Kopecek J. Angewandte Makromol Chem 1978, 70, 109.

18. Charlionet, R.; Machour-Merlet, N.; Leclerc, S.; Malandain, J. Electrophoresis 1997, 18, 1133.

19. Woerly, S.; Plant, G. W.; Harvey, A. R. Neurosci Lett 1996, 205, 197.

20. Charlionet, R.; Levasseur, L.; Melandarin, J. J. Electrophoresis 1996, 17, 58.

21. Zhang, X. Z.; Yang, Y. Y.; Chung, T. S.; Ma, K. X. Langmuir 2001, 17, 6094.

22. Zhang, W.; Liu, Y.; Zhu, M.; Zhang, Y.; Liu, X.; Yu, H.; Jiang, Y.; Chen, Y.; Kuckling, D.; Alder, H. J. P. J Polym Sci Part A: Polym Chem 2006, 44,6640 .

23. Woerly, S.; Pinet, E.; de Robertis, L.; Van Diep, D.; Bousmina, M. Biomaterials 2001, 22, 1095.

24. Woerly, S.; Doan, V. D.; Sosa, N.; de Vellis, J.; Espinosa-Jeffrey, A. J Neurosci Res 2004, 75, 262.

25. Woerly, S.; Awosika, O.; Zhao, P.; Agbo, C.; GomezPinilla, F.; de Vellis, J.; Espinosa-Jeffrey, A. Neurochem Res 2005, 30, 721. 